\title{
Greater Suppression of Glucagon, Lipolysis, and Ketogenesis with Insulin Glargine U300 as Compared with Glargine U100 in Type 1 Diabetes Mellitus
}

\author{
Paola Lucidi, MD, PhD, Francesca Porcellati, MD, PhD, Patrizia Cioli, RD, PhD, \\ Paola Candeloro, PhD, Anna Marinelli Andreoli, MD, PhD, \\ Geremia B. Bolli, MD, and Carmine G. Fanelli, MD, PhD
}

\begin{abstract}
The aim of this study was to establish the effects of clinical doses of Gla-300 versus Gla-100 on suppression of glucagon, lipolysis, and ketogenesis in type 1 diabetes mellitus (T1DM). Eighteen persons with T1DM (age $40 \pm 12$ years, diabetes duration $26 \pm 12$ years, body mass index $23.4 \pm 2 \mathrm{~kg} / \mathrm{m}^{2}$, A1C $7.19 \% \pm 0.52 \%$ [55 $\pm 6 \mathrm{mmol} / \mathrm{mol}]$ ) were studied after 3 months of titration with Gla-300 and Gla-100 (randomized, crossover design) with a 24-h euglycemic clamp (s.c. injection of individual insulin daily doses used by subjects for previous 2 weeks, Gla-300 $0.35 \pm 0.08$ and Gla-100 $0.28 \pm 0.07 \mathrm{U} / \mathrm{kg}$ ). Gla-300 resulted in (1) less increase in insulin concentration for $0-12 \mathrm{~h}$, but greater insulin concentration in 12-24h (no differences for $24 \mathrm{~h}$ ); (2) greater glucagon suppression; (3) greater prehepatic insulin-to-glucagon molar ratio, primarily in $12-24 \mathrm{~h}$ (ratio $1.78,90 \%$ confidence intervals [CIs] $1.5-2.1)$; and (4) lower $24-\mathrm{h}$ free fatty acid $(0.81 ; 90 \%$ CI $0.73-0.89$ ), glycerol $(0.78 ; 90 \%$ CI $0.65-0.94)$, and $\beta$-hydroxybutyrate $(0.72 ; 90 \%$ CI $0.58-0.90)$. Over the $24 \mathrm{~h}$ postinjection, as compared with Gla-100, clinical doses of Gla-300 exhibit greater suppressive effects on glucagon, lipolysis, and ketogenesis, whereas the effects on glucose metabolism are equivalent.
\end{abstract}

Keywords: Lipolysis, Ketogenesis, Glucagon, Glargine U300, Type 1 diabetes.

\section{Introduction}

$\mathbf{R}$ ECENT STUDIES HAVE compared the pharmacokinetics (PK) and pharmacodynamics (PD) of the long-acting (basal) insulin analogues glargine U300 (Gla-300) and glargine U100 (Gla-100) in type 1 diabetes mellitus (T1DM) at fixed insulin doses after single injection, ${ }^{1}$ at steady state,${ }^{2}$ and at individual doses that persons with T1DM require daily. ${ }^{3}$

In addition to glucose metabolism, insulin regulates several physiological pathways, such as modulation of glucagon secretion, ${ }^{4}$ effects on lipid ${ }^{5}$ and protein $^{6}$ metabolism, and endothelial function. ${ }^{7}$ Only one study, investigating PK/PD of glargine and detemir at steady state in T1DM, however, has reported on the effects of long-acting insulin analogues, on regulation of alanine, lactate, and lipid metabolism.
The aim of this study was to establish the potential differences between clinical doses of Gla-300 and Gla-100 on suppression of glucagon, lipolysis, and ketogenesis, in a group of persons with T1DM in whom the results of glucose metabolism have already been described. ${ }^{3}$

\section{Materials and Methods}

Subjects, study design, and methods have been previously described in detail. ${ }^{3}$ In brief, 18 persons with T1DM (age $40 \pm 12$ years, diabetes duration $26 \pm 12$ years, body mass index $23.4 \pm 2 \mathrm{~kg} / \mathrm{m}^{2}$, A1C $7.19 \% \pm 0.52 \%$ [55 $\pm 6 \mathrm{mmol} /$ mol], plasma C-peptide $0.01 \pm 0.01 \mathrm{ng} / \mathrm{mL}$ ) were studied after 3 months of optimization of glycemic control with Gla-300 or Gla-100 (evening dosing), titrated to fasting

Section of Endocrinology \& Metabolism, Department of Medicine, Perugia University School of Medicine, Perugia, Italy.

Preliminary data from this study were submitted to the 77th Scientific Sessions (2017), June 9-13, 2017, San Diego, California, and has been accepted for poster presentation with abstract publication in Diabetes. 
near-normoglycemia (90-110 mg/dL), in addition to prandial rapid-acting insulin analogues (randomized, crossover study with 2 months washout period).

Subjects were studied during $24 \mathrm{~h}$ fasting, with the euglycemic glucose clamp at steady state of basal insulin treatment, after s.c. injection at $20.00 \mathrm{~h}$ of the clinical dose of Gla-300 and Gla-100 that they were on in everyday life. The study treatment was open, but blind to clamp investigators. Bedside plasma glucose (PG) and serum-free insulin concentration (FIRI) were measured by previously described methods. ${ }^{3}$ Plasma glucagon was measured by a commercial radioimmunoassay kit (DRG Instruments $\mathrm{GmbH}$, Marburg, Germany): blood glycerol, $\beta$-hydroxybutyrate, alanine, and lactate by previously described fluorimetric methods. ${ }^{9}$ Free fatty acid (FFA) values were measured using a commercial kit (Wako NEFA C test kit; Wako Chemicals, Neuss, Germany). All parameters were measured every $2 \mathrm{~h}$. The study protocol was approved by the Ethical Study Committee of Umbria Region (CEAS) and registered with EudraCT number 2015-002135-17.

The endpoint was the area under the plasma glucagon, blood FFA, glycerol, and $\beta$-hydroxybutyrate concentration curves (AUC) for $24 \mathrm{~h}$, and in the second versus first 12-h postdosing time intervals with Gla-300 versus Gla-100. Another endpoint was prehepatic insulin-to-glucagon molar ratio.

\section{Calculations and Statistical Methods}

The linear trapezoidal rule was used to calculate the AUC of the parameters measured. The prehepatic insulin-toglucagon molar ratio was calculated from the peripheral concentrations of insulin (which equals portal in the absence of endogenous secretion) and glucagon (corrected for hepatic clearance), as previously reported. ${ }^{10}$ Fluctuation of prehepatic insulin-to-glucagon molar ratio around the average value was calculated as [(insulin-to-glucagon molar ratio $\mathrm{C}_{\max }$ - insulin-to-glucagon molar ratio $\mathrm{C}_{\min }$ /insulin-toglucagon molar ratio $\mathrm{C}_{\mathrm{avg}}$ )]. Point estimates of treatment ratios (Gla-300/Gla-100), with $90 \%$ confidence intervals (CIs), were calculated using ANOVA, which allowed for variation due to sequence, subjects nested within sequence, period, and treatment based on log-transformed data and retransformations. Equivalence was to be concluded if the $90 \%$ CIs for each parameter were completely contained within the interval 0.80-1.25. Data are expressed as mean \pm standard deviation or geometric means $(95 \% \mathrm{CI})$ as appropriate in tables and in text, and in figures as means and standard error. Statistical analysis was usually performed using NCSS12/ PASS11 (NCSS, LLC. Kaysville, UT).

\section{Results}

\section{Glycemic control before studies and PG concentration} in euglycemic clamp studies

Glycemic control before the studies was no different with Gla-300 versus Gla-100 (A1C 6.94 0.48 and $6.97 \% \pm$ $0.49 \%$ [52 \pm 5 and $53 \pm 6 \mathrm{mmol} / \mathrm{mol}]$, respectively), mean PG was no different, and hypoglycemia did not occur in the 3 days before studies. ${ }^{3}$ A modest amount of i.v. insulin and/or glucose was needed before the clamp (from -5 to $0 \mathrm{~h}$ ) to normalize $\mathrm{PG}^{3}{ }^{3}$ The total amount of insulin infused was lower $(0.76 \pm 0.55$ and $1.33 \pm 0.68 \mathrm{U}$ of insulin), whereas glucose infused was higher $(5.04 \pm 5.9$ and $0.79 \pm 1.01 \mathrm{~g}$ of glucose $)$ with Gla-300 versus Gla-100, respectively. ${ }^{3}$ PG was $100 \pm 1.2$ and $101 \pm 1.8 \mathrm{mg} / \mathrm{dL}$ during the $24 \mathrm{~h}$ clamp with Gla-300 and Gla-100, respectively. The quality of the performance of the clamp studies was quite good based on the coefficient of variation of PG measurements $3.5 \% \pm 1.3 \%$.

\section{Serum-free insulin, plasma glucagon, and prehepatic insulin-to-glucagon molar ratio}

The mean doses of basal insulin injected s.c. at T0 of the clamp were $0.35 \pm 0.08$ and $0.28 \pm 0.07 \mathrm{U} / \mathrm{kg}$ with Gla-300 and Gla-100, respectively (steady-state daily doses used by subjects over the previous 15 days).

Serum insulin concentration increased less with Gla-300 versus Gla-100 over the initial $6 \mathrm{~h}$, then it was comparable between 6 and $18 \mathrm{~h}$, and finally was more elevated for last $6 \mathrm{~h}$ of the study in the interval $18-24 \mathrm{~h}^{3}$ (Fig. 1 and Supplementary Table S1)

Baseline plasma glucagon at T0 was lower with Gla-300 (51 pg/mL, 44-58) versus Gla-100 (62 pg/mL, 56-62), Gla300/Gla-100 ratio 0.82 (CIs 0.74-0.91). After s.c. insulin injection, plasma glucagon was similarly suppressed with both Gla-300 and Gla-100 for the first $12 \mathrm{~h}$ of study, but more so in the second $12 \mathrm{~h}$ postdosing with glucagon AUC $19 \%$ (13\%-24\%) lower with Gla-300 versus Gla-100. (Fig. 1 and Supplementary Table S1)

The prehepatic insulin-glucagon molar ratio at $\mathrm{T} 0$ was $70 \%$ (39\%-109\%) higher with Gla-300 versus Gla-100 (4.1, CIs 3.2-5.4 and 2.4, CIs $1.7-3.4 \mathrm{~mol} / \mathrm{mol}$, respectively). The prehepatic insulin-glucagon molar ratio increased to similar values with both insulins during the first $12 \mathrm{~h}$, although the relative increase was lower with Gla-300. In the second $12 \mathrm{~h}$ of study, the prehepatic insulin-glucagon molar ratio remained more stable with Gla-300 and was 78\% (50\%-109\%) greater than Gla-100. (Fig. 1 and Supplementary Table S1) Fluctuation of the prehepatic insulin-glucagon molar ratio was $37 \%$ lower with Gla-300 as compared with that with Gla-100.

\section{FFA, glycerol, and $\beta$-hydroxybutyrate}

At T0, with Gla-300 versus Gla-100, FFA concentrations were $40 \%(46 \%-78 \%)$ lower $(244,200-290$ and 390, 320$0.490 \mu \mathrm{mol} / \mathrm{L}$, respectively), $\beta$-hydroxybutyrate $70 \%$ (46\%$83 \%)$ lower $(60,40-90$ and $190,140-270 \mu \mathrm{mol} / \mathrm{L}$, respectively), despite lower rates of i.v. insulin infusion over the $5 \mathrm{~h}$ before study with Gla-300 versus Gla-100. ${ }^{3}$ After s.c. injection of insulin, FFA values with the two insulins were similar until $12 \mathrm{~h}$ postdosing, after which time the increase in FFA was greater with Gla-100 versus Gla-300 until the end of the study. FFA values were $24 \%$ (14\%-32\%) lower at $24 \mathrm{~h}$ postinjection of Gla-300 versus Gla-100 (Fig. 2 and Supplementary Fig. S1 and Supplementary Table S1). A similar $24 \mathrm{~h}$ pattern was observed with glycerol. (Fig. 2 and Supplementary Fig. S1 and Supplementary Table S1). $\beta$-Hydroxybutyrate suppressed more within 2-4 h after s.c. injection of Gla-100, thus mimicking the pattern of FFA, and remained suppressed until $16 \mathrm{~h}$. Thereafter, $\beta$-hydroxybutyrate increased more with Gla-100 until $24 \mathrm{~h}$. (Fig. 2 and Supplementary Fig. S1 and Supplementary Table S1).

The overall suppressive effect on lipolysis and ketogenesis of both insulins, as assessed by the averaged ratio of FFA, glycerol, and $\beta$-hydroxybutyrate, was $21 \%, 15 \%$, and $23 \%$ greater during $24 \mathrm{~h}(0-24 \mathrm{~h})$, during the first $12 \mathrm{~h}(0-12 \mathrm{~h})$, 

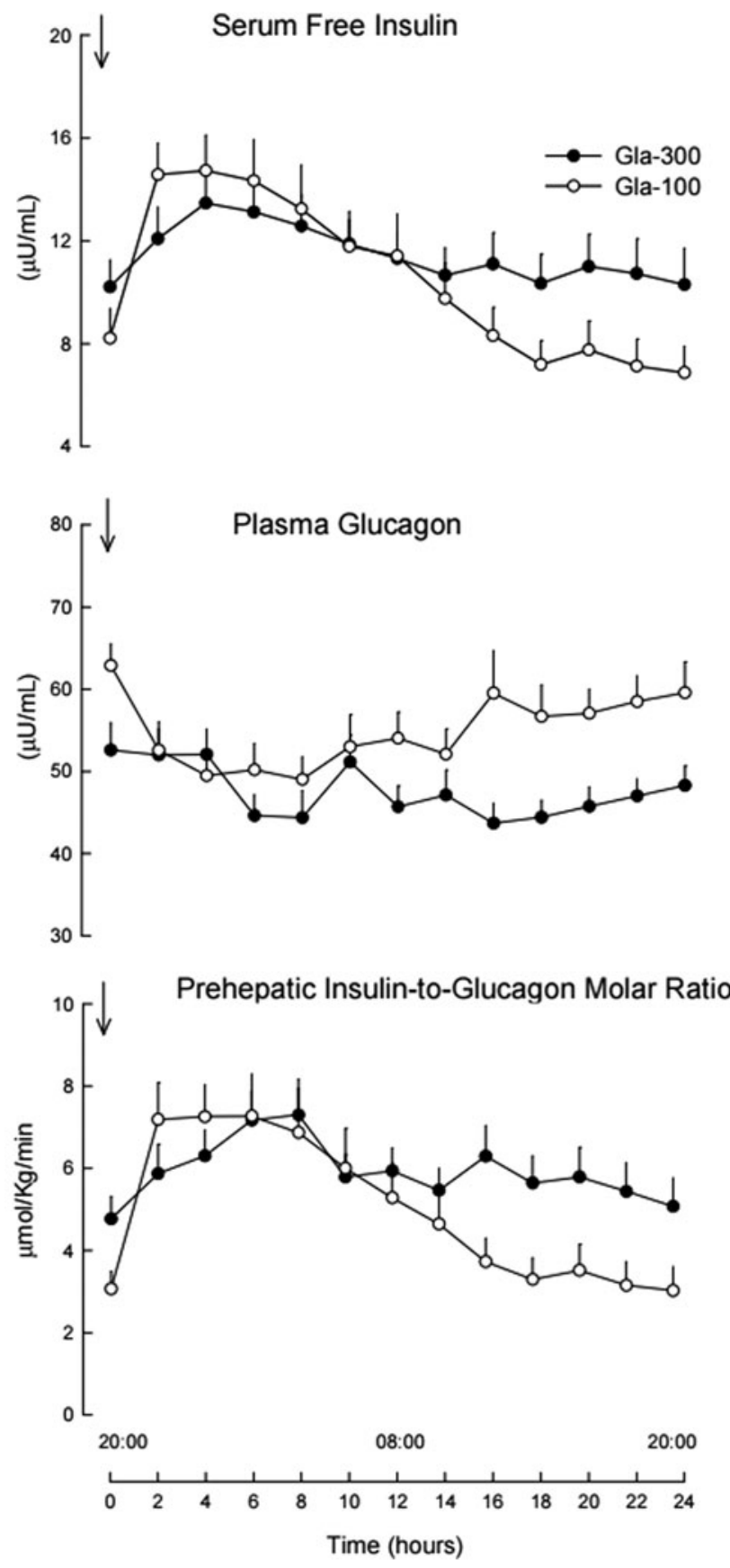

FIG. 1. Serum-(free) insulin and plasma glucagon concentrations and prehepatic insulin-to-glucagon molar ratio during $24 \mathrm{~h}$ postinjection of clinical individual doses of basal insulin Gla-300 (full dots) and Gla-100 (open dots) in 18 persons with T1DM. T1DM, type 1 diabetes mellitus.

and during the last $12 \mathrm{~h}(12-24 \mathrm{~h})$, respectively, with Gla-300 as compared with Gla-100 (Supplementary Fig. S1). The changes of FFA, $\beta$-hydroxybutyrate, and glucagon over the $24 \mathrm{~h}$ with Gla-300 were lower as compared with Gla-100 as directly measured by the treatment $\times$ time interaction term in the repeated measures ANOVA (two-way repeated-measures ANOVA) (treatment $\times$ time, $F 17,272=2.93, P=0.033$ [FFA]; $F 17,272=2.96,0.021[\beta$-hydroxybutyrate $]$ and $F 17,272=4.2$, $P=0.002$ [glucagon], all with Greenhouse-Geisser correction).

\section{Alanine and lactate}

Alanine and lactate concentrations were equivalent, with absolute values lower by $\sim 10 \%$ with Gla-300 versus Gla- 100 . (Supplementary Fig. S2 and Supplementary Table S1).

\section{Discussion}

This study demonstrates that clinical individual doses of insulin Gla-300 suppress glucagon and lipid metabolism more than Gla-100 doses over the $24 \mathrm{~h}$ postdosing. This result is new and is at variance with the effects observed on glucose metabolism that are equivalent for the $24 \mathrm{~h}$ with the two insulins. $^{3}$

The effects of Gla-300 versus Gla-100 here reported on glucagon and lipid metabolism are explained primarily by the differences in serum insulin dynamics between the two insulins. With Gla-300 serum insulin tends to be lower in first $12 \mathrm{~h}$, but is higher in second $12 \mathrm{~h}$ postdosing (Fig. 1 and Supplementary Table S1). This translates into greater suppression of glucagon and lipid metabolism, primarily in second $12 \mathrm{~h}$ postdosing with Gla-300 versus Gla-100 (Figs. 1 and 2 and Supplementary Table S1). Under these experimental conditions, also glucose metabolism was more stimulated in the second $12 \mathrm{~h}$ postdosing with Gla-300 versus Gla-100, but interestingly the overall $24 \mathrm{~h}$ effect of the two insulins was similar. $^{3}$ Thus, there are differences between glucose ${ }^{3}$ and lipid metabolism (present study), which may be explained by the well-known greater insulin sensitivity of adipose tissue versus liver glucose metabolism. This has been shown for minimal increments in serum insulin concentration in normal nondiabetic subjects, ${ }^{5,11}$ and, likely, this is the case also in persons with T1DM on fair glycemic control, as were those of this study.

The insulin doses of Gla-300 used in this study by the T1DM persons were higher than those of Gla-100, however, resulting in similar glycemic control (A1C) as well as equivalent effects on glucose metabolism over the $24 \mathrm{~h}^{3}{ }^{3}$ This is consistent with previous clinical trials wherein glycemic control was matched with the two insulins used at different doses $^{12}$ and may be explained by the need to compensate for the lower bioavailability of Gla-300 versus Gla- 100 after s.c. administration. ${ }^{2,13}$

The greater suppression of glucagon with Gla-300 versus Gla-100 was modest, as modest were the differences in insulin concentrations over the $24 \mathrm{~h}$. Under these conditions, the noninvasively estimated prehepatic insulin-to-glucagon molar ratio is a more useful parameter than isolated individual concentrations of serum insulin and glucagon, to interpret liver glucose metabolism (Fig. 1). ${ }^{10}$ In fact, it is the relative concentration of the two islet hormones that perfuse the liver through the portal route, to determine the fine physiological tuning of Endogenous Glucose Production, more than isolated individual concentrations of serum insulin and glucagon. ${ }^{10}$

The greater suppression of glucagon and lipid metabolism with Gla-300 versus Gla-100 appears quantitatively small and one might wonder whether the magnitude of the difference would be clinically significant to patient care. Clearly, that would require a larger study with clinical outcomes. Indeed, this PK/PD study provides a rational basis for such a study.

It is tempting to speculate on the possible beneficial effects of second-generation long-acting insulins with more robust 

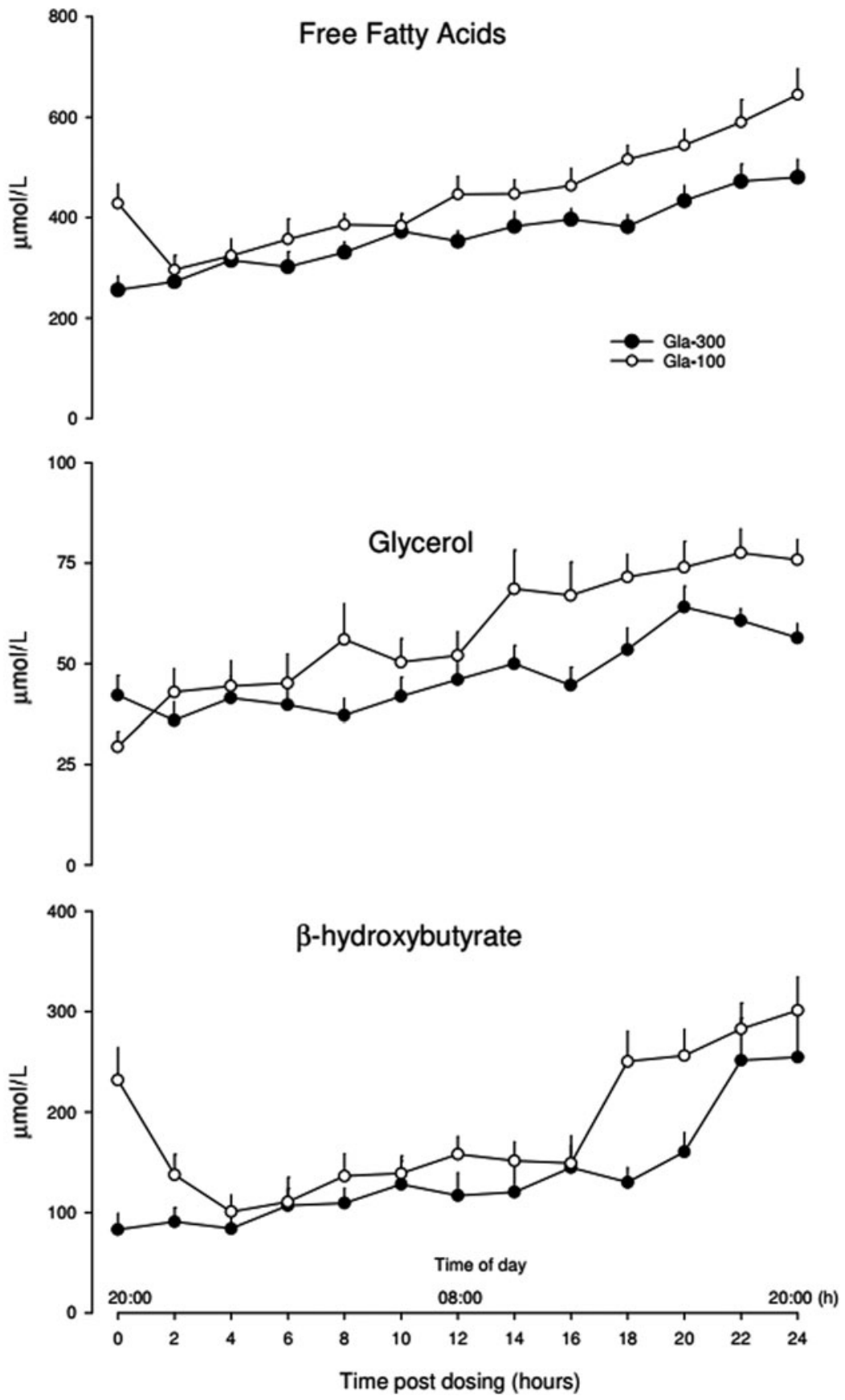

FIG. 2. Blood FFA, glycerol, and $\beta$ hydroxybutyrate concentrations during $24 \mathrm{~h}$ postinjection of clinical individual doses of basal insulin Gla-300 (full dots) and Gla-100 (open dots) in 18 persons with T1DM. FFA, free fatty acid. suppression of glucagon and lipid metabolism, like Gla-300 for people with T1DM. With evening dosing of Gla-300, the greater estimated prehepatic insulin-to-glucagon molar ratio accounts for lower EGP $24 \mathrm{~h}$ postdosing, ${ }^{3}$ which could explain the predinner lower glucose value observed in Continuous Glucose Monitoring studies. ${ }^{14,15}$ The greater lipid and ketone body suppression of Gla-300 over the $24 \mathrm{~h}$ might help reducing the risk of Diabetic Ketoacidosis in some T1DM people presenting recurrent DKA on Gla-100 or detemir, but ad hoc studies are required to prove this hypothesis. In this regard, it will be interesting to see the results of the ongoing trial with a similar question with insulin degludec versus Gla-100 and detemir. ${ }^{16}$

In conclusion, the greater suppressive effects of clinical doses of Gla-300 on glucagon, and lipid metabolism, add to the differences shown in $\mathrm{PK} / \mathrm{PD},{ }^{3}$ and help in understanding the potentially more beneficial effects of Gla-300 versus Gla100 in people with T1DM.

\section{Acknowledgments}

The authors are grateful to the persons with T1DM who have volunteered for the study for free (in Italy no payment/ reimbursement for volunteers of clinical research is allowed). Clinical Trial Information: EudraCT 2015-002135-17.

\section{Authors' Contributions}

P.L. enrolled patients, performed clamps, analyzed data, reviewed/edited the article; F.P. enrolled patients, performed clamps, analyzed data, wrote the clinical protocol, and 
reviewed/edited the article; P. Candeloro performed clamps, laboratory assays, and reviewed/edited the article; P.C. performed clamps and reviewed/edited the article; A.M.A. performed clamps and reviewed/edited the article; C.G.F. enrolled patients, performed clamps, analyzed data, performed statistical analysis, and wrote the article; and G.B.B. provided the study concept and design, supervised the protocol development and the research, enrolled patients, and wrote the article. C.G.F. is the guarantor of this study and, as such, had full access to all the data in the study and takes responsibility for the integrity of the data and the accuracy of data analysis.

\section{Author Disclosure Statement}

P.L. has received travel grants for scientific meetings from Sanofi, Novo, Abbott, and Menarini, F.P. has received honoraria for lectures and consultations from Sanofi, Eli Lilly, and Astra Zeneca, and travel grants from Sanofi; P.C., P.C., and A.M.A. disclose no conflict of interest. G.B.B. has received honoraria for lectures and consultations from Sanofi and Menarini; C.G.F. has received honoraria for lecturing and consultations from Sanofi and travel grants from Menarini.

\section{Funding Information}

The study is an investigator initiated trial, partially funded by Sanofi.

\section{Supplementary Material}

Supplementary Figure S1

Supplementary Figure S2

Supplementary Table S1

\section{References}

1. Shiramoto M, Eto T, Irie S, et al.: Single-dose new insulin glargine $300 \mathrm{U} / \mathrm{ml}$ provides prolonged, stable glycaemic control in Japanese and European people with type 1 diabetes. Diabetes Obes Metab 2015;17:254-260.

2. Becker RH, Dahmen R, Bergmann K, et al.: New insulin glargine 300 Units $\mathrm{mL}^{-1}$ provides a more even activity profile and prolonged glycemic control at steady state compared with insulin glargine 100 Units $\mathrm{mL}^{-1}$. Diabetes Care 2015;38:637-643.

3. Porcellati F, Lucidi P, Candeloro P, et al.: Pharmacokinetics, pharmacodynamics, and modulation of hepatic glucose production with insulin Glargine U-300 and Glargine U-100 at steady-state, individualized clinical doses in type 1 diabetes mellitus. Diabetes Care 2019;42: 85-92.

4. Gosmanov NR, Gosmanov AR, Gerich JE: Glucagon physiology. In: De Groot LJ, Chrousos G, Dungan K, Feingold KR, Grossman A, Hershman JM, Koch C, Korbonits M, McLachlan R, New M, Purnell J, Rebar R, Singer F, Vinik A, eds. Endotext [Internet]. South Dartmouth, MA: MDText.com, Inc., 2000-2011.

5. Nurjhan N, Campbell PJ, Kennedy FP, et al.: Insulin doseresponse characteristics for suppression of glycerol release and conversion to glucose in humans. Diabetes 1986;35: 1326-1331.

6. De Feo P, Gaisano MG, Haymond MW: Differential minimals of insulin deficiency on albumin and fibrinogen synthesis in humans. J Clin Invest 1991;88:833-840.

7. Chaudhuri A, Dandona P, Fonseca V: Cardiovascular benefits of exogenous insulin. J Clin Endocrinol Metab 2012;97:3079-3091.

8. Porcellati F, Rossetti P, Busciantella NR, et al.: Comparison of pharmacokinetics and dynamics of the long-acting insulin analogs glargine and detemir at steady state in type 1 diabetes: a double-blind, randomized, crossover study. Diabetes Care 2007;30:2447-2452.

9. Fanelli CG, De Feo P, Porcellati F, et al.: Adrenergic mechanisms contribute to the late phase of hypoglycemic glucose counterregulation in humans by stimulating lipolysis. J Clin Invest 1992;89:2005-2013.

10. Ferrannini E, Muscelli E, Frascerra S, et al.: Metabolic response to sodium-glucose cotransporter 2 inhibition in type 2 diabetic patients. J Clin Invest 2014;124:499-508.

11. Gerich JE: Physiology of glucose homeostasis. Diabetes Obes Metab 2000;2:345-350.

12. Home PD, Bergenstal RM, Bolli GB, et al.: Glycaemic control and hypoglycaemia during 12 months of randomized treatment with insulin glargine $300 \mathrm{U} / \mathrm{mL}$ versus glargine $100 \mathrm{U} / \mathrm{mL}$ in people with type 1 diabetes (EDITION 4). Diabetes Obes Metab 2018;20:121-128.

13. Werner U, Korn M, Tennagels N: Insulin glargine $300 \mathrm{U} / \mathrm{ml}$ and insulin glargine $100 \mathrm{U} / \mathrm{ml}$ show equipotent in vivo blood glucose lowering when administered intravenously in dogs (abstract). Diabetes Technol Ther 2016;18:A-119.

14. Bergenstal RM, Bailey TS, Rodbard D, et al.: Comparison of Insulin Glargine 300 Units $/ \mathrm{mL}$ and 100 Units $/ \mathrm{mL}$ in adults with Type 1 Diabetes: continuous glucose monitoring profiles and variability using morning or evening Injections. Diabetes Care 2017;40:554-560.

15. Pettus J, Gill J, Paranjape S, et al.: Efficacy and safety of a morning injection of insulin glargine 300 units $/ \mathrm{mL}$ versus insulin glargine 100 units $/ \mathrm{mL}$ in adult patients with type 1 diabetes: a multicentre, randomized controlled trial using continuous glucose monitoring. Diabetes Obes Metab 2019. [Epub ahead of print]; DOI: 10.1111/dom.13751.

16. ClinicalTrials.gov [Internet]. Bethesda (MD): National Library of Medicine (US). Identifier NCT03001323, Insulin Degludec for the Management of Patient With Recurrent Diabetic Ketoacidosis; (Last Update Posted : April 16, 2019); Available from: https://www.clinicaltrials.gov/ct2/ show/study/NCT03001323

Address correspondence to: Carmine G. Fanelli, $M D, P h D$ Section of Endocrinology \& Metabolism Department of Medicine Perugia University School of Medicine Hospital Santa Maria della Misericordia Torre Ellittica, Floor +1 , Room \#17 Perugia 06129 Italy

E-mail: carmine.fanelli@unipg.it 